\title{
High-intensity inspiratory muscle training in bronchiectasis: A randomized controlled trial
}

\author{
Ozge OZALP, ${ }^{1}$ Deniz INAL-INCE, ${ }^{1}$ (D) Aslihan CAKMAK, ${ }^{1}$ Ebru CALIK-KUTUKCU, ${ }^{1}$ Melda SAGLAM, ${ }^{1}$ \\ Sema SAVCI, ${ }^{2}$ NaCIYE VARDAR-YAGLI, ${ }^{1}$ HÜlya ARIKAN, ${ }^{1}$ Jale KARAKAYA ${ }^{3}$ AND LÜTFI COPLU ${ }^{4}$ \\ ${ }^{1}$ Faculty of Health Sciences, Department of Physiotherapy and Rehabilitation, Hacettepe University, Ankara, Turkey; ${ }^{2}$ School \\ of Physiotherapy and Rehabilitation, Dokuz Eylül University, Izmir, Turkey; ${ }^{3}$ Faculty of Medicine, Department of Biostatistics, \\ Hacettepe University, Ankara, Turkey; ${ }^{4}$ Faculty of Medicine, Department of Chest Medicine, Hacettepe University, \\ Ankara, Turkey
}

\begin{abstract}
Background and objective: Inspiratory muscle training (IMT) enhances velocity of inspiratory muscle contraction and modifies inspiratory and expiratory time. This study aimed to examine the impact of high-intensity IMT (H-IMT) on exercise capacity in bronchiectasis.

Methods: Forty-five patients were included. Lung function, respiratory muscle strength and endurance, exercise capacity, dyspnoea, fatigue and quality of life (QOL) were evaluated. Patients were randomized into two groups: H-IMT and control groups. Twenty-three patients underwent H-IMT for 8 weeks, using threshold loading with a target workload of maximal inspiratory pressure (MIP) of at least $70 \%$, with 3 -min cycles (as 2-min training: 1-min rest intervals) for $21 \mathrm{~min}$. There was a total period of $14 \mathrm{~min}$ of loaded breathing and $7 \mathrm{~min}$ of recovery. The control group $(n=22)$ underwent low-intensity IMT at $10 \%$ of the initial MIP and was maintained at the same intensity until the end of the training.

Results: After training, both MIP and maximal expiratory pressure (MEP) and the incremental shuttle walk distance were increased in the H-IMT group compared with the control group $(P<0.05)$. There was a significant difference in constant threshold load, time and pressure-time units in the H-IMT group $(P<0.05)$ but not in the control group $(P>0.05)$. A significant decrease was found in fatigue in both groups $(P<0.05)$. The Leicester Cough Questionnaire social score for the H-IMT group decreased significantly after the treatment $(P<0.05)$.

Conclusion: The H-IMT increased exercise capacity in patients with non-cystic fibrosis bronchiectasis. It has also positive effects on respiratory muscle strength and endurance, and social aspects of QOL.
\end{abstract}

Clinical trial registration: NCT02656992 at ClinicalTrials.gov

Correspondence: Deniz Inal-Ince, Faculty of Health Sciences, Department of Physiotherapy and Rehabilitation, Hacettepe University, 06100 Samanpazari, Ankara, Turkey. Email: dince@ hacettepe.edu.tr

Received 3 April 2018; invited to revise 17 April, 24 May and 23 July 2018; revised 30 April, 22 June and 24 July 2018; accepted 8 August 2018 (Associate Editor: Conroy Wong; Senior Editor: Paul King).

\section{SUMMARY AT A GLANCE}

The present randomized controlled study contributes to the literature about the impact of highintensity inspiratory muscle training (H-IMT) in bronchiectasis. We showed that H-IMT increased respiratory muscle strength and endurance, exercise capacity and social aspects of quality of life in patients with non-cystic fibrosis bronchiectasis

Key words: bronchiectasis, exercise and pulmonary rehabilitation, quality of life, respiratory function tests.

Abbreviations: $\int P d t$, pressure-time unit; $6 \mathrm{MWT}, 6$-min walk test; BSI, Bronchiectasis Severity Index; CF, cystic fibrosis; EM, Expectation Maximization; ExT, exercise training; $\mathrm{FEF}_{25-75 \%}$, forced expiratory volume 25-75\%; $F E V_{1}$, forced expiratory volume in $1 \mathrm{~s}$; FSS, Fatigue Severity Scale; FVC, forced vital capacity; H-IMT, high-intensity IMT; HR, heart rate; HRmax, maximal HR; IMT, inspiratory muscle training; ISWT, incremental shuttle walk test; LCQ, Leicester Cough Questionnaire; mBS, modified Borg Scale; MEP, maximal expiratory pressure; MIP, maximal inspiratory pressure; MMRC, modified Medical Research Council; PEF, peak expiratory flow rate; PFT, pulmonary function test; $\mathrm{QOL}$, quality of life; $R R$, respiratory rate; SGRQ, St George Respiratory Questionnaire; $\mathrm{SIP}$, sustainable inspiratory pressure; $\mathrm{SpO}_{2}$, oxygen saturation.

\section{INTRODUCTION}

Bronchiectasis is a chronic lung disease with major symptoms of cough, excessive secretions, dyspnoea, exercise intolerance and fatigue. ${ }^{1,2}$

Inspiratory muscle weakness may lead to muscle load and capacity discordance, and thereby, dyspnoea, decreased exercise tolerance, hypoventilation and respiratory failure. A decrease in expiratory muscle strength impairs the effectiveness of coughing and decreases the removal of airway secretions. ${ }^{3,4}$

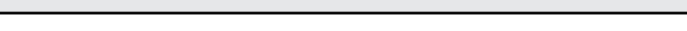


weakness. ${ }^{6}$ Inspiratory muscle training (IMT) using a threshold device can enhance velocity of inspiratory muscle contraction, decreased inspiratory time and increase exhalation time and allow more time for lung emptying. ${ }^{5}$ In bronchiectasis, one study combining IMT with whole-body exercise training (ExT) revealed that IMT has no additional effects on maximum inspiratory pressure (MIP), whereas the impact of ExT was sustained. ${ }^{1}$ The IMT also improved both inspiratory and expiratory muscle strengths without any impact on pulmonary function, quality of life (QOL) or exercise capacity. ${ }^{5}$

Interval-based high-intensity IMT (H-IMT) studies have been shown to provide more respiratory muscle function improvement than low-to-medium-intensity loads. ${ }^{7,8}$ Care to avoid fatigue by not applying H-IMT more than 3 days a week is a time-saving application compared to other methods.

As studies investigating the effects of IMT in bronchiectasis are limited, we aimed to examine the impact of isolated respiratory muscle training on significant physiological and clinical findings in bronchiectasis. Therefore, the purpose of this study was to explore the effects of H-IMT and low-IMT on exercise capacity in patients with clinically stable non-cystic fibrosis (CF) bronchiectasis. The hypothesis was that the $\mathrm{H}$ IMT would cause increased exercise capacity in stable bronchiectasis.

\section{METHODS}

\section{Subjects}

Clinically stable patients with non-CF bronchiectasis aged 18-65 years were included from June 2013 to June 2016. Bronchiectasis was confirmed by clinical history, including coughing, shortness of breath, exertional dyspnoea, pulmonary function tests (PFT) and highresolution computed tomography (HRCT). Subjects, clinically stable without any evidence of an exacerbation or changes in medical therapy in the previous 3 weeks, were randomly divided into two groups based on a computer programme: H-IMT and low-intensity IMT (control). Assessors for testing were blinded to the groups. Inclusion criteria were no significant coexisting disease affecting ability to undertake exercise, ability to walk and willingness to cooperate in the study. Patients with neurological complications, advanced orthopaedic disease, advanced heart failure, acute exacerbations in the last 3 weeks, and patients on antibiotics and previously participated in a rehabilitation programme were excluded. Hacettepe University Ethics Committee approved the study (12.06.2013, GO 13/292), which is registered at ClinicalTrials.gov (NCT02656992). All subjects gave their written informed consent to participate in the study. The primary outcome measure was incremental shuttle walk test (ISWT) distance. The power analysis was performed (G*Power Ver. 3.0.10; Franz Faul, Universität Kiel, Germany) based on two-way repeated-measures of analysis of variance. With a $>27.8-\mathrm{m}$ difference in the ISWT distance which is the difference between sham and intervention in the study by Newall et al., ${ }^{1} 80 \%$ power, $5 \%$ type I error and an effect size of $55 \%, 22$ patients in each group were needed for sufficient sample size. The trial was ended after reaching sufficient sample size.

\section{Measurements}

Demographic and physical characteristics, smoking history and symptoms were recorded. The HRCT findings were documented. Bronchiectasis Severity Index (BSI) score was calculated. ${ }^{9}$ All measurements were performed at the beginning and end of the 8-week programme by a blinded assessor to group allocation.

The PFT was performed using a standard spirometer (Spirodoc; MIR, Rome, Italy) and expressed as a percentage of the predicted values. ${ }^{10}$ Dyspnoea and fatigue were evaluated using the modified Medical Research Council (MMRC) dyspnoea scale ${ }^{11}$ and Fatigue Severity Scale (FSS), ${ }^{12}$ respectively.

Respiratory muscle strength (MIP and maximal expiratory pressure, MEP) was measured using an electronic mouth pressure device (Micro MPM; Micro Medical Ltd., Rochester, UK). The MIP and MEP were recorded as $\mathrm{cm} \mathrm{H}_{2} \mathrm{O}$ and expressed as the percent predicted values. ${ }^{13}$ Respiratory muscle endurance was measured with a threshold loading device (Powerbreathe, POWERbreathe International Ltd., Warwickshire, England), using the sustainable inspiratory pressure (SIP) test. The load was adjusted to $60 \%$ of MIP, and the duration of the trial was recorded. ${ }^{14}$ Subjects performed respiratory efforts while breathing through the threshold loading device's rubber tubing for $10 \mathrm{~min}$ with a lip seal as the nose was occluded. With the use of a sustained breathing frequency of 12-14 bpm with 15-min rest intervals between two SIP tests, and taking the better of the two tests, the effects of learning and breathing technique were minimized. Heart rate $(\mathrm{HR})$, oxygen saturation $\left(\mathrm{SpO}_{2}\right)$, respiratory rate (RR) and dyspnoea using the modified Borg Scale (mBS) were recorded before and after the test. The $\mathrm{mBS}$ is a 10-point categorical scale of dyspnoea on exertion. $^{15}$

Exercise capacity was evaluated using the ISWT. The test was ended if the patient reached maximal HR, $\mathrm{SpO}_{2}<80 \%$ and excessive dyspnoea, fatigue or pain. HR was evaluated using a polar HR monitor (Polar S610i; Polar Electro Oy, Kempele, Finland). $\mathrm{SpO}_{2}, \mathrm{RR}$ and dyspnoea (mBS) were assessed before and after the test. ${ }^{16}$ The distance was expressed both as metres and a percentage of the predicted distance. ${ }^{17}$ Minimal clinically significant difference for the ISWT is $35 \mathrm{~m}^{18}$

The QOL was measured using the Leicester Cough Questionnaire (LCQ). The scores range from 3 to 21, and higher scores indicate more impaired QOL from a cough; the questionnaire consists of physical, psychological and social dimensions. ${ }^{19}$

\section{Inspiratory muscle training}

The patients underwent H-IMT for 3 days/week for 8 weeks. One session/week was performed under the supervision, and the other two sessions/week were performed at home. A threshold loading device (Powerbreathe, POWERbreathe International Ltd., Warwickshire, England) was used to ensure inspiratory muscle loading. The device was previously used in 
bronchiectasis patients. ${ }^{1}$ For the first session, the target workload was selected at $30 \%$ of MIP. The H-IMT group underwent IMT with a 1-min warm-up on an inspiratory load of $15 \%$ of MIP. Then, the study and rest ratio of 2:1 progressed to $30 \%$ MIP within interval IMT. No training was given during the 1 -min rest period. Three-minute cycles were repeated seven times, and each session lasted $21 \mathrm{~min} .{ }^{7,20}$ Following the third session, inspiratory muscle load was targeted to be at least $70 \%$ of the MIP. There was a total period of 14 min of loaded breathing and $7 \mathrm{~min}$ of recovery. The load was further increased over the 8-week period with the aim of titrating to a level where subjects were just able to complete the final 2 -min interval. ${ }^{8}$ In addition, a rating of perceived exertion according to the $\mathrm{mBS}$ score of 3-5 was taken in determining target workload at the end of each 2-min working period. The control group underwent low-intensity IMT at $10 \%$ of the initial MIP and kept the same load until the end of the training. In each group, patients were adapted to their selected patterns of breathing. ${ }^{7,20}$ No instructions were given to the subject regarding what breathing pattern to adopt. In each session, $\mathrm{HR}$ and $\mathrm{SpO}_{2}$ were measured using a pulse oximeter (Nonin Palmsat 2550A, Noninedical Inc., Plymouth, MN, USA), and dyspnoea and rate of perceived exertion were evaluated using the mBS. Training workload was presented as the percentage of initial MIP value and pressure-time unit ( $\left.\int \mathrm{Pdt}\right) .^{7,20}$

Tolerability was assessed using the maximum workload achieved during the $\mathrm{H}-\mathrm{IMT}^{8}$ and $\mathrm{HR}, \mathrm{SpO}_{2}$, sensation of dyspnoea and perception of respiratory effort during progressive inspiratory loading. ${ }^{21}$

\section{Statistical analysis}

Statistical analysis was performed using SPSS Statistics (Version 23.0, IBM Inc., Armonk, NY, USA). An intention-to-treat analysis was performed. The Expectation Maximization (EM) method was used to overcome missing cases (two from H-IMT and one from control). The EM method is an iterative method using maximum likelihood of estimates. ${ }^{22}$ Descriptive statistics were calculated. Normality of the data was checked using the Kolmogorov-Smirnov test. Two independent groups of numerical variables were analysed using Student's ttest or the Mann-Whitney U-test, as appropriate. The chi-square test was used for categorical comparisons. Baseline values were compared using Student's t-test. Two-way analysis of variance was used to evaluate time and group-time interaction. Repeated measures of variance was used to analyse the data in each session. The descriptive level of significance was set at $P<0.05$.

\section{RESULTS}

Forty-five subjects with clinically stable bronchiectasis participated. One patient from the H-IMT group discontinued IMT due to an acute exacerbation and one patient due to inability to cooperate with the device. One patient from the control group stopped lowintensity IMT without giving any specific reason (Fig. 1).
Subjects were similar regarding age, gender, physical characteristics, smoking history, disease duration, aetiology of bronchiectasis, the number of acute exacerbations in the previous year, FSS and BSI scores $(P>0.05$; Table 1). The BSI scores indicated low to intermediate in terms of severity. The PFT values respiratory muscle strength and endurance were not different between the groups $(P>0.05$; Table 1$)$. The number of hospitalizations was higher in the H-IMT group $(P=0.047)$. The MMRC scores of the H-IMT group were significantly lower than those of the control group $(P<0.05)$. Initial exercise $\mathrm{HR}$, dyspnoea, fatigue and ISWT distance and LCQ scores except social scores $(P<0.05)$ were similar between the groups $(P>0.05$; Table 1). During the first week of training, initial MIP workload and $\int \mathrm{Pdt}$ of the H-IMT group were $50.32 \pm 10.06 \%$ and $6017.79 \pm 1711.19 \mathrm{~cm} \mathrm{H}_{2} \mathrm{O} . \mathrm{s}$ and of the control group were $9.91 \pm 0.33 \%$ and $1131.82 \pm 357.27 \mathrm{~cm} \mathrm{H}_{2} \mathrm{O} . \mathrm{s}$, respectively $(P<0.001)$. The workload increased to $98.31 \pm 15.83 \%$ (11 845.53 $\pm 3663.58 \mathrm{~cm} \mathrm{H}_{2} \mathrm{O} . \mathrm{s}$ ) in the H-IMT group, and the change from the initial workload at 8 weeks was statistically significant $(P<0.05)$. Achieving $98 \%$ of MIP during training and no significant change in HR and $\mathrm{SpO}_{2}$ during progressive loading $(P>0.05)$ indicated that H-IMT was well tolerated. The change in ISWT distance and the percent distance in the H-IMT group were significant compared to controls $(P<0.05$; Table 2). There was no change in PFT and MMRC in either the H-IMT or the control groups $(P>0.05$; Table 2). A significant decrease in FSS was found in both groups $(P<0.05)$ with no difference between the groups $(P>0.05$; Table 2$)$. The changes in MIP, MIP\%, MEP and MEP\% were significantly higher in the H-IMT group $(P<0.001$; Table 2$)$. There was a significant difference in SIP, time and $\int \mathrm{Pdt}$ values in the H-IMT group $(P<0.05$; Table 2$)$. The LCQ social scores for the $\mathrm{H}$-IMT group increased significantly after the treatment $(P<0.05$; Table 2).

\section{DISCUSSION}

On the basis of the findings, an 8-week H-IMT increased exercise capacity in non-CF patients with bronchiectasis with low to intermediate severity. The H-IMT has improved respiratory muscle strength and endurance, and social aspects of the QOL. The study protocol was well tolerated.

Exercise capacity diminishes in bronchiectasis due to ventilatory changes, gas exchange problems, respiratory mechanics, cardiovascular disorders, musculoskeletal changes and psychological factors. ${ }^{2,23}$ We evaluated exercise capacity using the ISWT. At baseline, $69.57 \%$ of patients from the H-IMT group and $63.64 \%$ of patients from the control group were at lower than the percent predicted values of ISWT, stating a reduced exercise capacity. In two studies investigating the effects of IMT on exercise capacity in bronchiectasis, IMT was applied as $30-60 \%$ of MIP, 30 min per day, 5-7 days per week for 8 weeks. In one study, ${ }^{1}$ the lack of an exclusive IMT group and application as a combination of ExT and IMT make comparison difficult. In the second study, 6-min walk test (6MWT) distance 


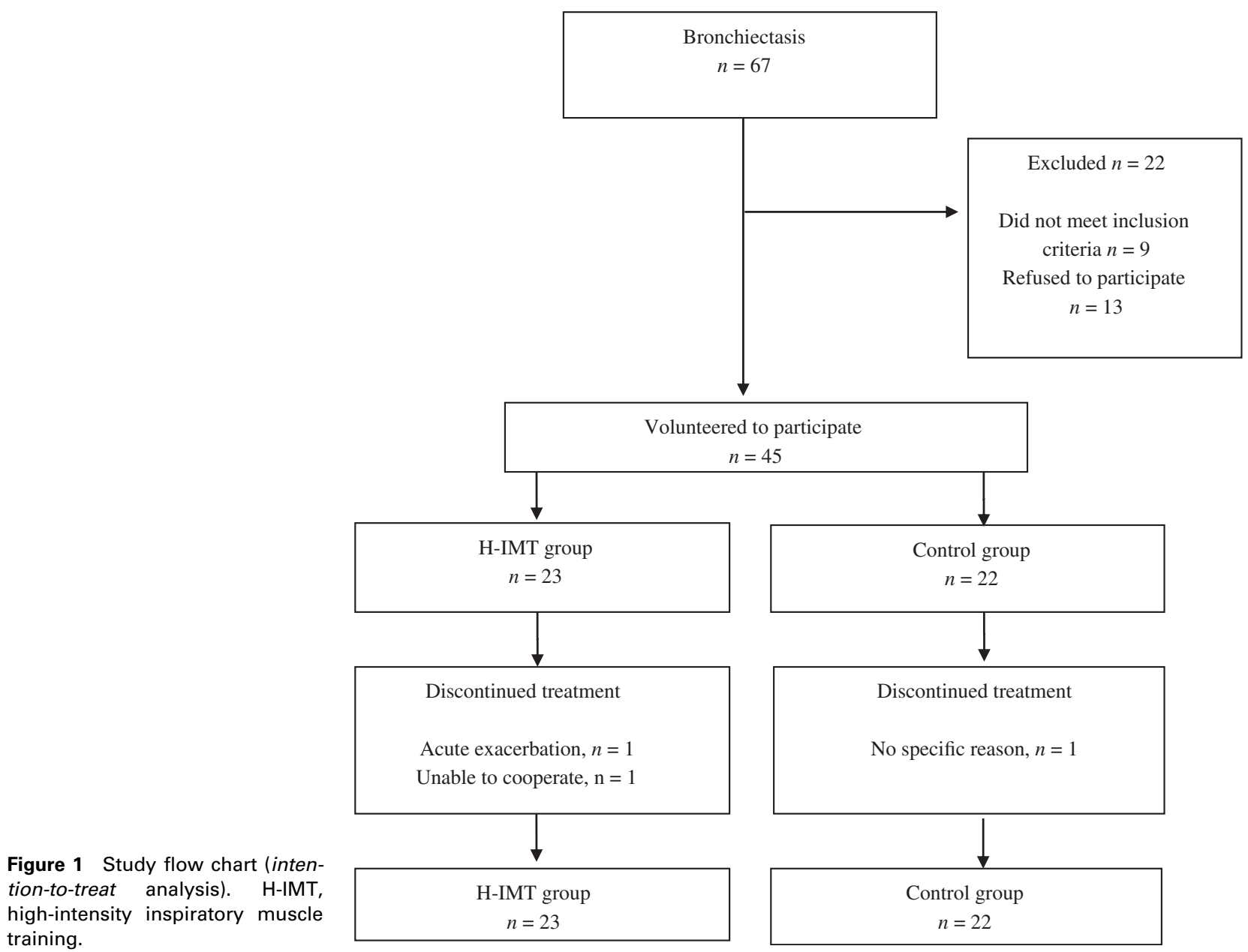

improved $61 \mathrm{~m}(14.89 \%)$ in the IMT group versus $20 \mathrm{~m}$ $(4.69 \%)$ in the control group. Although not statistically significant, the baseline ISWT for the H-IMT group was $53 \mathrm{~m}$ further than the control group which is a randomization effect. ${ }^{24}$ After treatment, we found a $50.3-\mathrm{m}$ $(7.19 \%)$ increase in the IMT group, which is above the minimal clinically significant difference. ${ }^{18}$ However, there was a small change in the control group $(0.2 \mathrm{~m}$ and $1.01 \%)$. The difference might be due to different characteristics of $6 \mathrm{MWT}$ versus ISWT. ${ }^{16,17}$ Other reasons may include differences in patients' age range, pulmonary function, respiratory muscle strength and IMT protocols between the studies.

Although not statistically significant, forced expiratory volume in $1 \mathrm{~s}\left(\mathrm{FEV}_{1}\right) \%$ predicted was $12 \%$ worse in the intervention arm. We also found no change in PFT and dyspnoea after H-IMT in patients with bronchiectasis, similar to the previous studies using IMT. ${ }^{5,8}$ The smoking history was minimal which is similar to the previous findings in patients with bronchiectasis. $^{25}$ The findings may reveal that H-IMT does not affect expiratory airflow and breathlessness in mild lung function impairment. Chronic fatigue is frequent in bronchiectasis and may affect exercise capacity and QOL. ${ }^{26}$ Finding no change in fatigue may be due to having a different sample size or using the FSS, which is not a questionnaire specific to lung disease.
Two studies investigated IMT in bronchiectasis. ${ }^{1,5}$ In the first study, an 8-week moderate-intensity (MIP $30-60 \%, 15 \mathrm{~min}$ ) IMT added to ExT did not show an increase in MIP. ${ }^{1}$ In the second study, which had low statistical power, a programme of low-intensity IMT (MIP $30 \%$ ), MIP and MEP improved by $39 \%$ and $44 \%$, respectively, after 8 weeks. In our study, H-IMT increased MIP and MEP by $43.53 \%$ and $11.67 \%$, respectively. The H-IMT protocol used in our study ensures achieving higher workloads without increasing dyspnoea perception. ${ }^{8}$ Inspiration against resistance during IMT may increase the activation of the expiratory muscles by the last force extension, which may result in a significant increase in MEP. Further study is needed to clarify the mechanism of H-IMT increasing respiratory muscle function.

Respiratory muscle endurance reflects the sustained performance of a given workload. ${ }^{3}$ No study has investigated respiratory muscle endurance during IMT. In our study, respiratory muscle endurance improved by $207 \mathrm{~s}(139 \%)$ in H-IMT and by $38 \mathrm{~s}(22 \%)$ in the control group, which were lower than the value of $261 \mathrm{~s}$ improvement in a meta-analysis. ${ }^{27}$ The differences in clinical characteristics of diseases and the study protocol may be responsible for this discrepancy. The $\mathrm{H}-$ IMT was a high-intensity interval exercise with frequent short rest periods and resulted in the improved endurance of respiratory muscles without causing 
Table 1 Characteristics of the subjects

\begin{tabular}{|c|c|c|c|c|}
\hline Characteristics & Total $(n=45)$ & $\mathrm{H}-\mathrm{IMT}(n=23)$ & Control $(n=22)$ & $P$-value \\
\hline Age (years) & $44.04 \pm 12.89$ & $42.22 \pm 14.30$ & $45.95 \pm 11.26$ & 0.337 \\
\hline Gender (female/male) & $33 / 12$ & $16 / 7$ & $17 / 5$ & 0.805 \\
\hline Height $(\mathrm{cm})$ & $162.36 \pm 7.36$ & $162.91 \pm 6.61$ & $161.77 \pm 8.19$ & 0.609 \\
\hline Weight (kg) & $68.64 \pm 14.41$ & $67.17 \pm 11.93$ & $70.18 \pm 16.76$ & 0.490 \\
\hline BMI $\left(\mathrm{kg} / \mathrm{m}^{2}\right)$ & $26.07 \pm 5.38$ & $25.41 \pm 4.94$ & $26.79 \pm 5.84$ & 0.396 \\
\hline Smoking (pack-years) & $0(0-30)$ & $0(0-25)$ & $0(0-30)$ & 0.850 \\
\hline Disease duration (years) & $7(1-40)$ & $6(1-40)$ & $8(1-35)$ & 0.460 \\
\hline \multicolumn{5}{|l|}{ Aetiology, $n(\%)$} \\
\hline Childhood infection & $27(60)$ & $14(60.9)$ & $13(59.1)$ & \\
\hline Infection & $6(13.3)$ & $3(13.0)$ & $3(13.6)$ & \\
\hline Tuberculosis & $5(11.1)$ & $3(13.0)$ & $2(9.1)$ & 0.990 \\
\hline Idiopathic & $3(6.7)$ & $1(4.4)$ & $2(9.1)$ & \\
\hline Immune deficiency & $2(4.4)$ & $1(4.4)$ & $1(4.5)$ & \\
\hline Inflammatory disease & $2(4.4)$ & $1(4.4)$ & $1(4.5)$ & \\
\hline \multicolumn{5}{|l|}{ Hospitalization, $n(\%)$} \\
\hline One time & $10(22.2)$ & $7(30.4)$ & $3(13.6)$ & 0.114 \\
\hline Two times & $1(2.2)$ & $1(4.3)$ & 0 & \\
\hline Haemoptysis, $n(\%)$ & $8(17.8)$ & $7(30.4)$ & $1-(4.5)$ & $0.027 *$ \\
\hline $\mathrm{FEV}_{1}(\%)$ & $72.98 \pm 20.31$ & $66.35 \pm 17.45$ & $78.50 \pm 22.72$ & 0.050 \\
\hline FVC (\%) & $85.87 \pm 20.52$ & $81.91 \pm 15.72$ & $91.41 \pm 22.01$ & 0.102 \\
\hline $\mathrm{FEV}_{1} / \mathrm{FVC}$ & $86.82 \pm 16.08$ & $84.17 \pm 16.41$ & $89.59 \pm 15.63$ & 0.263 \\
\hline PEF (\%) & $70.042 \pm 24.19$ & $66.65 \pm 25.82$ & $74.50 \pm 22.44$ & 0.274 \\
\hline $\mathrm{FEF}_{25-75 \%}(\%)$ & $47.04 \pm 26.91$ & $42.00 \pm 23.21$ & $52.32 \pm 29.93$ & 0.202 \\
\hline BSI score & $4.82 \pm 3.61$ & $5.65 \pm 3.82$ & $3.95 \pm 3.23$ & 0.116 \\
\hline MMRC score & $1(0-3)$ & $1(0-3)$ & $1(0-3)$ & $0.031 *$ \\
\hline FSS score & $4.71 \pm 1.12$ & $4.57 \pm 1.10$ & $4.84 \pm 1.14$ & 0.426 \\
\hline $\mathrm{MIP}\left(\mathrm{cm} \mathrm{H} \mathrm{H}_{2} \mathrm{O}\right)$ & $97.38 \pm 24.15$ & $99.70 \pm 18.91$ & $94.95 \pm 28.90$ & 0.521 \\
\hline MIP\% & $107.88 \pm 27.67$ & $108.30 \pm 22.41$ & $107.44 \pm 32.83$ & 0.919 \\
\hline $\operatorname{MEP}\left(\mathrm{cm} \mathrm{H}_{2} \mathrm{O}\right)$ & $115.73 \pm 26.39$ & $121.87 \pm 23.97$ & $109.32 \pm 27.80$ & 0.112 \\
\hline MEP\% & $70.10 \pm 18.81$ & $73.37 \pm 20.78$ & $66.69 \pm 16.28$ & 0.238 \\
\hline Sustainable inspiratory pressure $\left(\mathrm{cm} \mathrm{H}_{2} \mathrm{O}\right)$ & $58.39 \pm 14.33$ & $59.71 \pm 10.82$ & $57.00 \pm 17.42$ & 0.532 \\
\hline Time $(s)$ & $159.48 \pm 163.14$ & $149.40 \pm 170.99$ & $170.02 \pm 157.83$ & 0.677 \\
\hline $\int \mathrm{Pdt}\left(\mathrm{cm} \mathrm{H} \mathrm{H}_{2} \mathrm{O} . \mathrm{s}\right)$ & $9624.90 \pm 10671.83$ & $8882.47 \pm 10447.93$ & $10401 \pm 11091.90$ & 0.639 \\
\hline Respiratory rate (bpm) & $15.29 \pm 1.93$ & $15.04 \pm 1.83$ & $15.55 \pm 2.03$ & 0.336 \\
\hline Dyspnoea & $1.72 \pm 1.44$ & $1.36 \pm 1.57$ & $2.10 \pm 1.20$ & 0.057 \\
\hline Fatigue & $2.06 \pm 1.26$ & $1.60 \pm 1.26$ & $2.53 \pm 1.57$ & $0.012 *$ \\
\hline ISWT distance (m) & $608.44 \pm 158.59$ & $634.35 \pm 174.33$ & $581.36 \pm 139.12$ & 0.267 \\
\hline Distance (\%) & $73.42 \pm 16.11$ & $72.93 \pm 12.76$ & $73.94 \pm 19.30$ & 0.838 \\
\hline HRmax (bpm) & $156.84 \pm 17.55$ & $156.96 \pm 19.10$ & $155.68 \pm 16.13$ & 0.669 \\
\hline $\operatorname{HRmax}(\%)$ & $89.24 \pm 8.53$ & $88.93 \pm 8.94$ & $89.55 \pm 8.28$ & 0.812 \\
\hline Dyspnoea & $3.64 \pm 2.22$ & $3.83 \pm 2.62$ & $3.45 \pm 1.74$ & 0.580 \\
\hline Fatigue & $3.63 \pm 2.28$ & $3.35 \pm 2.17$ & $3.93 \pm 2.60$ & 0.266 \\
\hline Leg fatigue & $4.09 \pm 2.40$ & $3.70 \pm 2.49$ & $4.50 \pm 2.28$ & 0.417 \\
\hline \multicolumn{5}{|l|}{ LCQ } \\
\hline Physical & $4.90 \pm 1.03$ & $4.74 \pm 0.94$ & $5.07 \pm 1.12$ & 0.291 \\
\hline Psychological & $4.81 \pm 1.07$ & $4.60 \pm 1.14$ & $5.03 \pm 0.97$ & 0.188 \\
\hline Social & $5.32 \pm 1.32$ & $4.87 \pm 1.21$ & $5.80 \pm 1.29$ & $0.017 *$ \\
\hline Total (3-21) & $15.08 \pm 3.21$ & $14.21 \pm 3.07$ & $15.98 \pm 3.18$ & 0.064 \\
\hline
\end{tabular}

$* P<0.05$

Statistically significant values are given in bold.

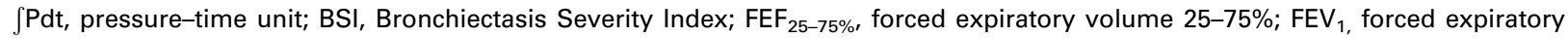
volume in $1 \mathrm{~s}$; FSS, Fatigue Severity Scale; FVC, forced vital capacity; H-IMT, high-intensity inspiratory muscle training; HRmax, maximal heart rate; ISWT, incremental shuttle walk test; LCQ, Leicester Cough Questionnaire; MEP, maximal expiratory pressure; MIP, maximal inspiratory pressure; MMRC, modified Medical Research Council; PEF, peak expiratory flow rate.

dyspnoea and fatigue. Whether H-IMT ensures cellular biochemical changes (increased type I fibres in the external intercostal muscles) in bronchiectasis, as in chronic obstructive pulmonary disease, ${ }^{28}$ is unclear.
The QOL assessment is essential to understand the effects of disease on patient's life and response to intervention. Health-related QOL is affected in bronchiecta$\mathrm{sis}^{29}$ due to the symptoms, the limitations in lung function and exercise tolerance. We evaluated QOL 


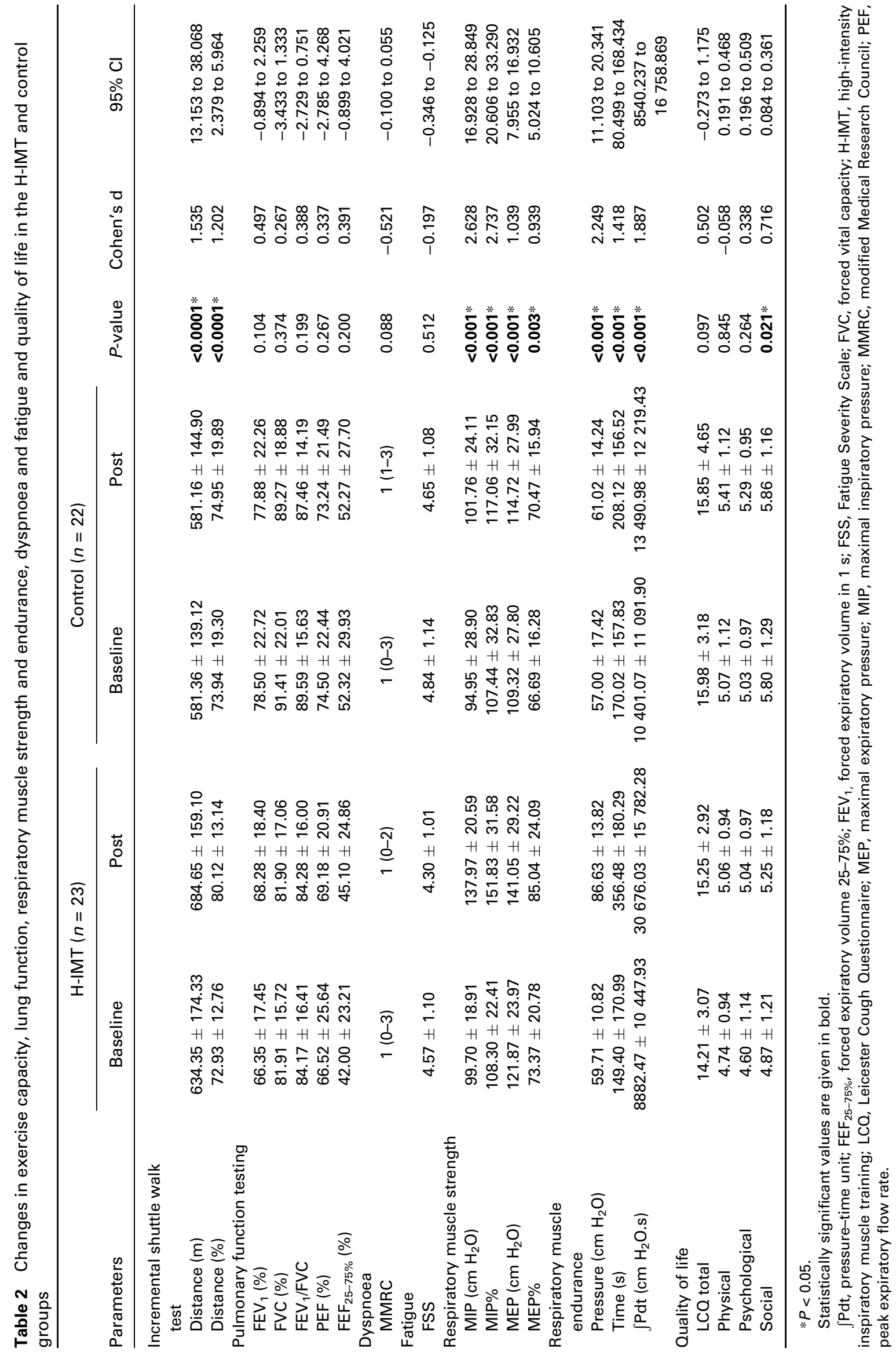


using the LCQ as the validation of other questionnaires such as St George Respiratory Questionnaire (SGRQ) has not been available in Turkish before the beginning of the study. We found social dimension scores were reduced in the H-IMT group at the beginning of the study, probably due to the effects of increased number of hospitalizations and the presence of haemoptysis, reflecting the severity of the disease. Two previous studies have investigated improved QOL, measured using the SGRQ, after IMT in bronchiectasis. ${ }^{1,5}$ One of those studies showed that the increase in QOL when compared with the other groups was similar to that of IMT given with ExT. It was revealed that the results were preserved in this group at the end of 3 months. In the other study, IMT was shown to increase the symptoms, activity and total scores compared with the control group. ${ }^{5}$ In our study, there was a similar increase in physical and psychological dimensions between the two groups and a significant increase in the social dimension. The aforementioned findings may be due to QOL as a subjective measurement or that the interval-based H-IMT does not make a difference regarding cough-specific QOL measured by the LCQ. In addition, the use of the LCQ which predominantly focuses on cough may limit the findings as compared with SGRQ which include other symptoms and their effects on activity.

In the present study, the H-IMT protocol was used to increase exercise capacity, respiratory muscle endurance and inspiratory muscle strength as well as dyspnoea in patients with bronchiectasis. Both the data in literature and those in our study suggest that H-IMT may be used in bronchiectasis patients as efficient to increase exercise capacity. The findings of our study revealed that respiratory muscles are capable of H-IMT, and it could be used as home-based. Thus, it may increase the patient's compliance and facilitate implementation for healthcare professionals. The limitations of the study were the number of patients included as we did not account for discontinuations, and the inability to perform the initial 2-week assessment period from the H-IMT protocol study which may act as a familiarization period. The aetiology of bronchiectasis is quite diverse reflecting common practice in bronchiectasis, and we checked group equivalence with the severity of illness using BSI. The patients in this study were relatively younger, thus further study is needed to investigate how this technique would be tolerated by an older group. The lung function seems to be very close to the normal values. The effects of H-IMT in patients with a worse lung function require further investigation. In conclusion, this is the first study to show that an interval-based H-IMT protocol is a reliable method for achieving adequate loading in bronchiectasis patients. The H-IMT increased exercise capacity besides respiratory muscle strength and endurance, and may be included in pulmonary rehabilitation programmes for bronchiectasis patients. Further study is needed-specifically, welldesigned, sufficiently powered, randomized controlled trials to compare H-IMT with the other components of pulmonary rehabilitation, such as peripheral muscle training and aerobic training in patients with bronchiectasis, to understand exercise tolerance, dyspnoea perception, fatigue perception and the effect on QOL.

\section{Data availability statement}

Data sharing is unavailable.

\section{REFERENCES}

1 Newall C, Stockley RA, Hill SL. Exercise training and inspiratory muscle training in patients with bronchiectasis. Thorax 2005; 60: 943-8.

2 Koulouris NG, Retsou S, Kosmas E, Dimakou K, Malagari K, Mantzikopoulos G, Koutsoukou A, Milic-Emili J, Jordanoglou J. Tidal expiratory flow limitation, dyspnoea and exercise capacity in patients with bilateral bronchiectasis. Eur. Respir. J. 2003; 21: 743-8.

3 Troosters T, Gosselink R, Decramer M. Respiratory muscle assessment. Eur. Respir. Mon. 2005; 31: 57-71.

4 Moran F, Piper A, Elborn JS, Bradley JM. Respiratory muscle pressure in non-CF bronchiectasis: repeatability and reliability. Chron. Respir. Dis. 2010; 7: 165-71.

5 Liaw MY, Wang YH, Tsai YC, Huang KT, Chang PW, Chen YC, Lin MC. Inspiratory muscle training in bronchiectasis patients: a prospective randomized controlled study. Clin. Rehabil. 2011; 25: 524-36.

6 O'Shea SD, Taylor NF, Paratz J. Peripheral muscle strength training in COPD: a systematic review. Chest 2004; 126: 903-14.

7 Sturdy G, Hillman D, Green D, Jenkins S, Cecins N, Eastwood P. Feasibility of high-intensity, interval-based respiratory muscle training in COPD. Chest 2003; 123: 142-50.

8 Hill K, Jenkins SC, Philippe DL, Cecins N, Shepherd KL, Green DJ, Hillman DR, Eastwood PR. High-intensity inspiratory muscle training in COPD. Eur. Respir. J. 2006; 27: 1119-28.

9 Chalmers JD, Goeminne P, Aliberti S, McDonnell MJ, Lonni S, Davidson J, Poppelwell L, Salih W, Pesci A, Dupont LJ et al. The bronchiectasis severity index: an international derivation and validation study. Am. J. Respir. Crit. Care Med. 2014; 189: 576-85.

10 Quanjer PH, Tammeling GJ, Cotes JE, Pedersen OF, Peslin R, Yernault JC. Lung volumes and forced ventilatory flows. Report Working Party Standardization of Lung Function Tests. European Community for Steel and Coal. Official Statement of the European Respiratory Society. Eur. Respir. J. Suppl. 1993; 16: 5-40.

11 Bestall J, Paul E, Garrod R, Garnham R, Jones PW, Wedzicha JA. Usefulness of the Medical Research Council (MRC) dyspnoea scale as a measurement of disability in patients with chronic obstructive pulmonary disease. Thorax 1999; 54: 581-6.

12 Armutlu K, Korkmaz N, Keser I, Sumbuloglu V, Akbiyik D, Guney Z, Karabudak R. The validity and reliability of the fatigue severity scale in Turkish multiple sclerosis patients. Int. J. Rehabil. Res. 2007; 30: 81-5.

13 Black LF, Hyatt RE. Maximal respiratory pressures: normal values and relationship to age and sex. Am. Rev. Respir. Dis. 1969; 99: 696-702.

14 Nickerson BG, Keens TG. Measuring ventilatory muscle endurance in humans as sustainable inspiratory pressure. J. Appl. Physiol. 1982; 52: 768-72.

15 Borg GA. Psychophysical bases of perceived exertion. Med. Sci. Sports Exerc. 1982; 14: 377-81.

16 Singh SJ, Morgan MDL, Scott S, Walters D, Hardman AE. Development of a shuttle walking test of disability in patients with chronic airways obstruction. Thorax 1992; 47: 1019-24.

17 Probst VS, Hernandes NA, Teixeira DC, Felcar JM, Mesquita RB, Goncalves CG. Reference values for the incremental shuttle walking test. Respir. Med. 2012; 106: 243-8.

18 Lee AL, Hill CJ, Cecins N, Jenkins S, McDonald CF, Burge AT, Rautela L, Stirling RG, Thompson PJ, Holland AE. Minimal 
important difference in field walking tests in non-cystic fibrosis bronchiectasis following exercise training. Respir. Med. 2014; 108 1303-9.

19 Kalpaklioglu AF, Kara T, Kurtipek E, Kocyigit P, Ekici A, Ekici M. Evaluation and impact of chronic cough: comparison of specific versus generic quality-of-life questionnaires. Ann. Allergy Asthma Immunol. 2005; 94: 581-5.

20 Hill K, Cecins NM, Eastwood PR, Jenkins SC. Inspiratory muscle training for patients with chronic obstructive pulmonary disease: a practical guide for clinicians. Arch. Phys. Med. Rehabil. 2010; 91: 1466-70.

21 Eastwood PR, Van Der Touw TJ, Sturdy GA, Jenkins SC, Hillman DR. Anaerobic metabolism of inspiratory muscles in COPD. Respirology 2006; 11: 32-40.

22 Dempster AP, Laird NM, Rubin DB. Maximum likelihood from incomplete data via the EM algorithm. J. R. Stat. Soc. Series B Methodol. 1977; 39: 1-38.

23 O'Leary CJ, Wilson CB, Hansell DM, Cole PJ, Wilson R, Jones PW. Relationship between psychological well-being and lung health status in patients with bronchiectasis. Respir. Med. 2002; 96: 686-92.

24 Altman DG, Doré CJ. Randomisation and baseline comparisons in clinical trials. Lancet 1990; 335: 149-53.
25 Gale NS, Bolton CE, Duckers JM, Enright S, Cockcroft JR, Shale DJ. Systemic comorbidities in bronchiectasis. Chron. Respir. Dis. 2012; 9: 231-8.

26 Bott J, Blumenthal S, Buxton M, Ellum S, Falconer C, Garrod R, Harvey A, Hughes T, Lincoln M, Mikelsons C et al.; British Thoracic Society Physiotherapy Guideline Development Group. Guidelines for the physiotherapy management of the adult, medical, spontaneously breathing patient. Thorax 2009; 64(Suppl. 1): i1-i51.

27 Gosselink R, De Vos J, van den Heuvel SP, Segers J, Decramer M, Kwakkel G. Impact of inspiratory muscle training in patients with COPD: what is the evidence? Eur. Respir. J. 2011; 37: 416-25.

28 Ramirez-Sarmiento A, Orozco-Levi M, Guell R, Barreiro E, Hernandez N, Mota S, Sangenis M, Broquetas JM, Casan P, Gea J. Inspiratory muscle training in patients with chronic obstructive pulmonary disease: structural adaptation and physiologic outcomes. Am. J. Respir. Crit. Care Med. 2002; 166: 1491-7.

29 Spinou A, Fragkos KC, Lee KK, Elston C, Siegert RJ, Loebinger MR, Wilson R, Garrod R, Birring SS. The validity of health-related quality of life questionnaires in bronchiectasis: a systematic review and meta-analysis. Thorax 2016; 71: 683-94. 